\title{
Automatic Segmentation of Multi-Beam Data for Predictive Mapping of Benthic Habitats on the Chella Seamount (North-Eastern Alboran Sea, Western Mediterranean)
}

\author{
Enrique Coiras, Claudio Lo Iacono, Eulalia Gràcia, Juanjo Dañobeitia, and José Luis Sanz
}

\begin{abstract}
A reliable and objective classification method has been produced for the differentiation of benthic habitats in the seamount regions of the North-Eastern Alboran Sea. Acoustic backscatter and depth measurements from multi-beam data are automatically fused and then classified using video transects of known cold-water coral ecosystems as ground-truth. Results of the classification reveal the locations of potentially similar habitats in the region, and could be used as a base map for the planning of future scientific campaigns in the area.
\end{abstract}

Index Terms-Acoustic imaging, automatic classification, benthic habitats, data fusion, marine geology.

\section{INTRODUCTION}

I $\mathrm{N}$ THE LAST decade, scientific and institutional communities have shown a growing interest in understanding and preserving marine habitats, a tendency that has resulted in an increase of scientific activity and in the establishment of new environmental policies to protect them. The habitat of an organism (or community of organisms) is the place where the organism (or community) naturally occurs, a location with a particular set of environmental conditions that favor its proliferation. In the marine environment, benthic habitats determine the distribution, abundance, and diversity of seabed communities. Unfortunately, benthic habitats are increasingly threatened by human impact, such as trawl fishing and marine pollution [1]-[3]. For this reason, it is important to acquire detailed information about them and their distribution, which can further facilitate policy making strategies for the protection of vulnerable and ecologically sensitive areas [4]. The growing interest in producing solid guidelines to standardize habitat mapping methods has resulted

Manuscript received August 2010; revised January 24, 2011; accepted March 01, 2011. This work was sponsored by NURC's Visiting Researcher Program. Surveys were funded through the EVENT National Project (CGL2006-12861C02-02) and the EVENT-SHELF Complementary Action (CTM2008-03346-E) of the Spanish Ministry of Science and Innovation (MICINN).

E. Coiras was with the NATO Undersea Research Centre (NURC), 19126 La Spezia, Italy until August 2010. He is now with the European Union Satellite Centre, 28850 Torrejon de Ardoz, Madrid, Spain (e-mail: e.coiras@eusc.europa.eu).

C. Lo Iacono, E. Gràcia, and J. Dañobeitia are with the Unidad de Tecnologia Marina (UTM), Consejo Superior de Investigaciones Cientificas (CSIC), 08083 Barcelona, Spain (e-mail: loiacono@utm.csic.es; egracia@utm.csic.es; jjdanobeitia@cmima.csic.es).

J. L. Sanz is with the Spanish Oceanographic Institute (IEO), 28002 Madrid, Spain (e-mail: josel.sanz@md.ieo.es).

Color versions of one or more of the figures in this paper are available online at http://ieeexplore.ieee.org.

Digital Object Identifier 10.1109/JSTARS.2011.2123874
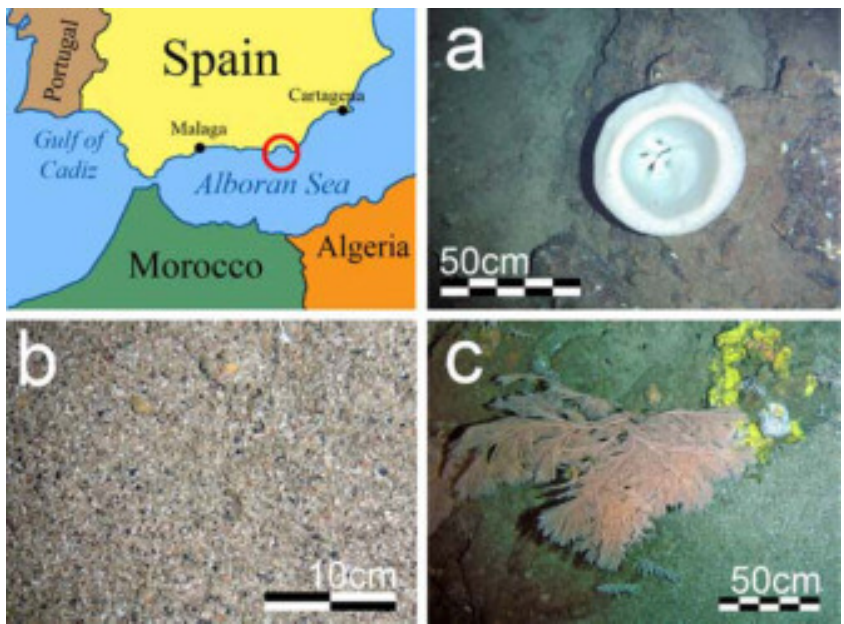

Fig. 1. Location of the surveyed area and main classes of interest: (a) hard rock outcrop showing a sponge, (b) detritic sand patch, and (c) Gorgonian assemblage on coarse terrain.

in many national (e.g.,: MAREMAP [5], INFOMAR [6]) and international projects (e.g.,: EC-MESH [7]). Methods to detect and classify benthic habitats rely mostly on acoustic sensors [8], [9] such as multi-beam and side-scan sonars. These sensors, however, will most often provide only indirect information, due to limits in resolution and because of variability in the measured acoustic returns. Several studies have recently attempted to automatically derive predictive maps of benthic habitats, such as the endangered Cold Water Corals' (CWC), based on comprehensive environmental approaches [10], [11] and on spatial and statistical analysis of seascape surrogates extracted from geo-acoustical data [12]-[15]. Special attention has been dedicated to the segmentation of backscatter images for habitat mapping based on their textural aspect and to angular signatures [16]-[18].

This paper presents data preprocessing and classification methods for multi-beam data and their application to habitat characterization in the Chella Seamount, North-Eastern Alboran Sea, Western Mediterranean (see map in Fig. 1). Our aim is to differentiate and map the most prevalent habitats observed in the area based on the local acoustic response and surface characteristics.

The main result of this study is a reliable and objective habitat classification method for the predictive mapping of benthic communities. 


\section{DATASET}

Swath-bathymetry of the Chella region was collected using the full water depth Simrad EM-300-a $30 \mathrm{kHz}$ multi-beam sonar with 135 independent beams-onboard the RV Vizconde de Eza [19]. Video transects in the region were acquired during the EVENT-Shelf survey (September 2009), carried out onboard the RV Garcia del Cid [20]. The remotely operated vehicle (ROV) Mariscope SPY-ICM was used for the shallowest transects $(100-200 \mathrm{~m})$, whereas the deep towed video-camera Seatronics DTS6000 was used in the deepest sectors of the Chella Bank (200-400 m). A 25 liter Van Veen grab was also deployed to collect sediment.

\section{ClassificATION}

For the automatic classification of the multi-beam sonar data we used a supervised approach [21]. The classifier was trained on multi-beam samples corresponding to locations where the video transects could serve as ground-truth to determine the class of interest. A linear classifier then extended the classification to the whole region covered by the multi-beam sonar. The Sections III-A-III-D describe this process in detail.

\section{A. Data Preprocessing and Fusion}

The geometry of multi-beam sonars determines the distribution of recorded data intensities. Since we are interested in classifying the seafloor according to its acoustic reflectivity, the influence of the observation direction must be removed or minimized as much as possible. Generally, Time-Varying Gain (TVG) methods compensate for most of the variability of the intensity profile caused by changes in range and incidence angle. This compensation is enough for qualitative data visualization, but for quatitative analysis a furhter corrections are required.

The most apparent intensity artifact in the multi-beam data was the decrease of the acoustic returns when moving from orthogonal incidence. Some of this variation is already compensated by the TVG but there is still some noticeable residual variation present in the data, as shown in Fig. 2(a). An additional hurdle is that the intensity profile depends on the roll coordinate of the multi-beam platform, which conditions what sonar beam has normal incidence with the seafloor. If the roll estimates are available, they can be used to drive the intensity compensation. Otherwise, as in our case, determination of the beam with normal incidence can be effected by estimating the path that maximizes the intensity returns:

$$
X=\arg \max _{\left\{x_{i}\right\}}\left(\sum_{i} I\left(i, x_{i}\right)\right) .
$$

Where $I$ is the backscatter intensity, $i$ is the line number and $x_{i}$ is the optimal selection of beam number for line $i$. The collection of the most normal beams for all lines is the optimal path $\mathrm{X}$, which can be found using dynamic programming [22]. A similar approach using principal component analysis was proposed in [23]. Fig. 2(a) shows a section of one of the data files and Fig. 2(b) the path most likely corresponding to the position of the beam with normal incidence, estimated with the proposed method.
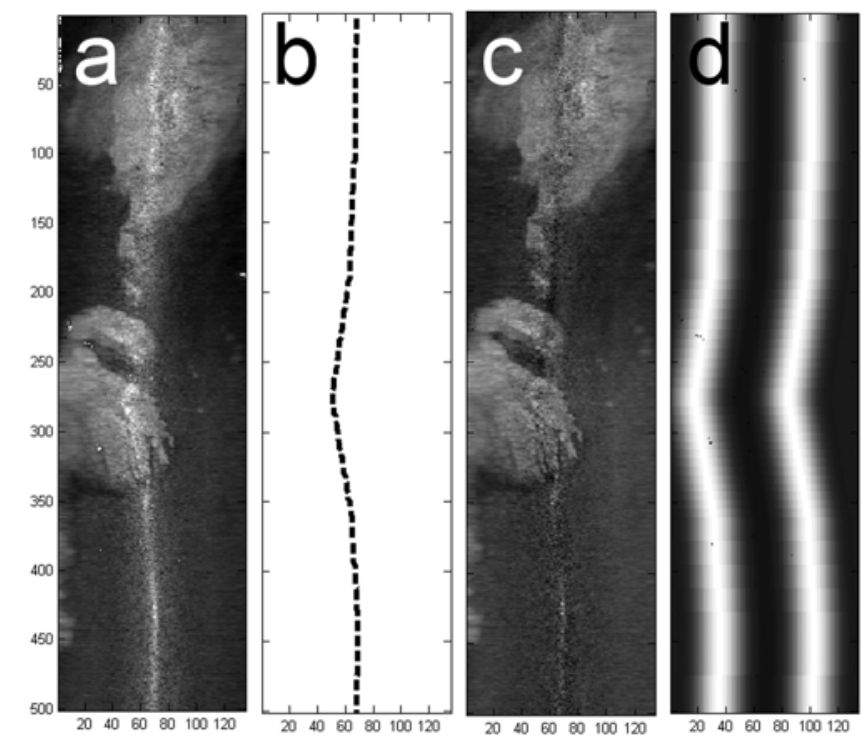

Fig. 2. Compensation of intensity variations with beam number and weighting function: (a) original backscatter image; (b) estimated location of the beam with normal incidence; (c) compensated backscatter image; (d) weight function for subsequent data fusion.

Once the beams with normal incidence are found, average intensity per incidence angle can be more accurately estimated and its variation discounted from the data. With this procedure we are basically making sure that seabed reflectivity is not affected by our angle of observation. As we are working in $\mathrm{dB}$, the compensation is done by subtracting the per-column average and restoring the global average $\langle I\rangle$ :

$$
I(i, j)=I(i, j)+\langle I\rangle-\frac{1}{N} \sum_{i} I\left(i, j-x_{i}+68\right) .
$$

Where 68 corresponds to the number of the default central beam (of the 135 beams the EM-300 has) and $N$ is the number of lines in the backscatter image.

The result of the preprocessing is shown in Fig. 2(c). For display purposes the residual intensity variations could be further reduced with more aggressive processing [24] but this would result in minor information losses - due to the substitution of noisy measured data values with extrapolated estimations based on texture synthesis from neighboring data points - that we would rather avoid for the current application.

In fact, the reliability of the samples is an important question when using multi-beam backscatter data. Typically, measures closer to normal incidence are noisier due to more prevalent specular effects while those at farther ranges have poorer resolution due to bigger beam footprints. This means that beams that minimize both incidence angle and range are the most reliable. Measurements from this loosely-defined medium range, usually called the "sweet spot", should therefore be valued higher. Finding an exact theoretical formula for the weight is complicated, since it involves the subjective decision of whether spatial resolution is more important than signal noise, and thus we have simply weighted the backscatter returns using a cubic spline with maximum weight for the sweet spot areas and minimum for farthest and normal incidence regions. Fig. 2(d) shows the 


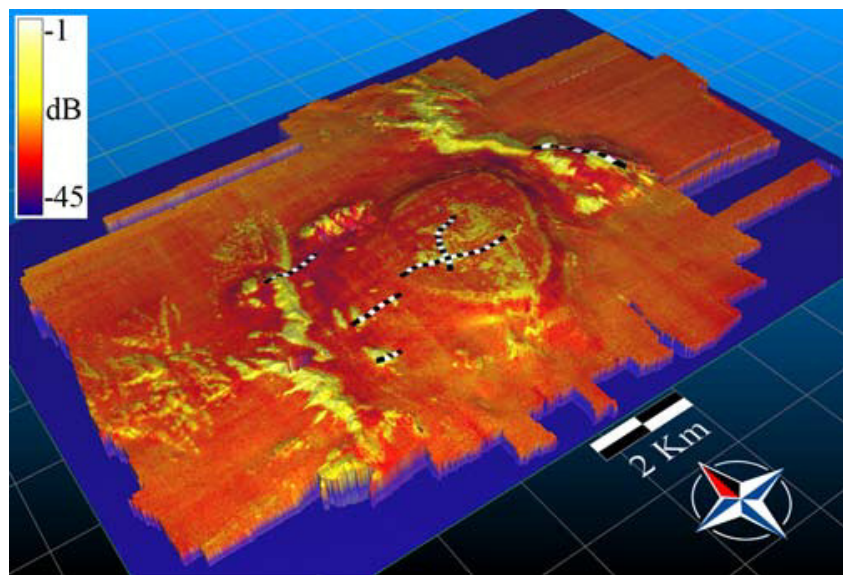

Fig. 3. Bathymetric map showing location of the video tracks used for groundtruthing. Texture of the depth map corresponds to backscatter data after fusion of all available multi-beam files. Only surface points that have been observed at least twice during the mission are shown.

weight function resulting from this approach once roll estimation using (1) has been considered.

After all data files were preprocessed, they were geo-referenced and fused by weighted averaging into the final backscatter and depth mosaics, resulting in two maps of 15,464 by 11,206 meters with a grid resolution of 2 meters.

In a further step to ensure only reliable data were used, those regions that after integration resulted in being observed only once were discarded. The final fused map is shown in Fig. 3, which displays the integrated depth mesh using the backscatter intensity as a texture. Brighter regions correspond to sections of the seabed with stronger reflectivities.

\section{B. Training Data and Classes}

Training data for the classifier was obtained from manual ground-truthing of the video collected by the ROV and the deep-towed camera. From the annotated tracks, the visited seafloor regions were set to one of the three following classes: "sand", "rock" and "gorgonian". This resulted in a ground-truthed training set of 368 samples (204 for the "sand" class, 101 for "rock" and 63 for "gorgonian").

The "sand" class corresponds to regions of medium to coarse sandy detritic seabed, with small blocks and scattered boulders. Analysis of their composition revealed the presence of planktonic foraminifera, shells, molluscs (especially bivalves), echinoid and bryozoans debris.

The "rock" class denotes areas where reliefs of hard and massive rock outcrops were observed. These regions, typically below $200 \mathrm{~m}$ depth, correspond to habitats mostly dominated by the presence of sponges: hat-shaped glass sponges and probably Phakellia ventilabrum. Spotty fine to medium sediment veneer were also found in these areas.

Finally, the most interesting "gorgonian" class groups those regions where deep-sea gorgonian assemblages (mostly Callogorgia verticillata, Viminella flaggellum) were found, typically on rough sub-horizontal outcrops mixed with very coarse sands. Small patches with stony CWC (Madrepora oculata) were also present in these areas. Video tracks showed that this habitat is seriously damaged by constant trawl fishing activities.

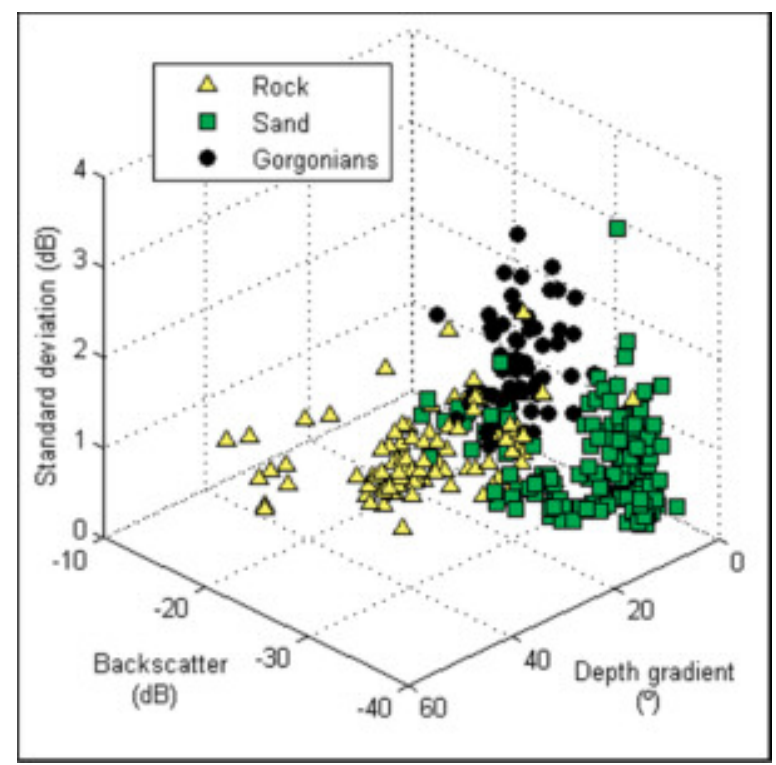

Fig. 4. Training samples from the ground-truth in feature space, showing the separability of the selected classes. Units are $\mathrm{dB}$ for the backscatter intensity and its standard deviation, and degrees for the slope variation.

\section{Features and Classification}

Direct observation of the fused depth and backscatter mosaics for the ground-truthed areas suggested that classification could be achieved with a limited number of features. In effect, "gorgonian" regions were characterized by strong returns showing a rich texture in the backscatter map, "sand" regions were typically flat with moderate backscatter returns, and "rock" regions showed steeper gradients and strong backscatter returns.

These observations permitted us to select just three features for the classification of the data: backscatter strength, local standard deviation of the backscatter (a basic image texture descriptor) and absolute value of the local depth gradient. A scatter plot of the ground-truth samples in this feature space (Fig. 4) clearly shows them to be separable. This validates our initial choice of features as they should allow for using simpler, more reliable, classification methods. It can also be noted from the figure that surface gradients play an important role in the differentiation of the rock and gorgonian classes, demonstrating the advantage that the bathymetric capability of multi-beam sensors has for this type of studies.

Classification with the selected features was performed using Matlab's "classify" routine, a linear classifier that fits a multivariate normal density to each class using a pooled estimate of covariance [25]. This resulted in the final class map shown in Fig. 5.

\section{Validation}

The classification approach was verified by 10 -fold cross-validation [26] of the ground-truthed training data, which resulted in a reasonable $12 \%$ classification error.

The limited number of features and the simplicity of the linear classifier used for description of the mission data suggest the classification approach is sound, limiting the likelihood of overfitting or other undesired classification artifacts. 


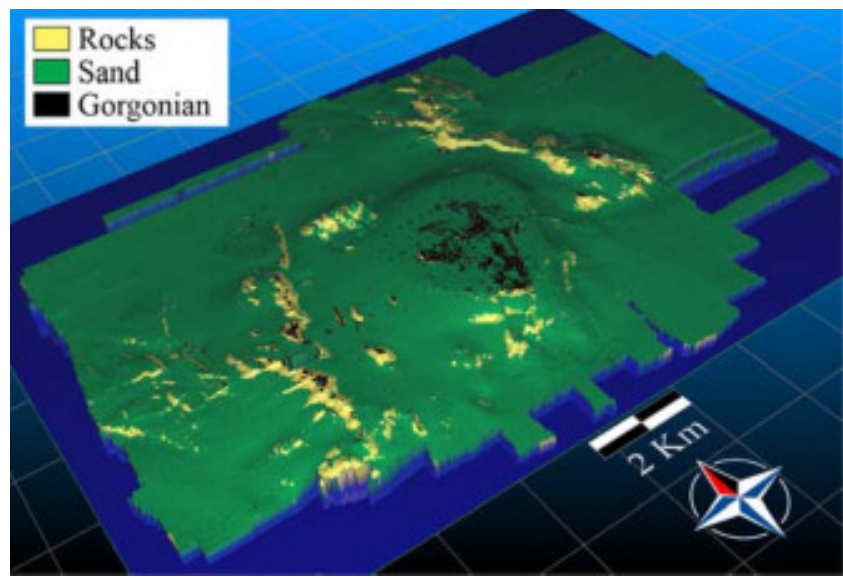

Fig. 5. Final class map, showing "rocks" in light yellow, "sand" in green, and "gorgonian" in black.

\section{RESUltS AND Discussion}

Segmentation of backscatter data and the resulting maps provide the identification of different and singular habitats on the Chella Bank. Gorgonians and related fauna at the top of the seamount between 100 and 130m (hard substrate, flat seafloor), scattered sponges on positive reliefs (hard substrate, deeply sloping) and coarse bioclastic sands plus epibenthic organisms in the sediments (soft substrate, flat or gently sloping). The higher texture values corresponding to the "gorgonian" class (Fig. 4) are probably due to the higher complexity of this habitat which develops on irregular and articulated rocky substrate and with a large associated fauna.

Classification results seem to indicate gorgonian assemblages tend to group at the flat top of the seamount between 100 and $120 \mathrm{~m}$, and on the top of several rocky ridges and peaks around the seamount (Fig. 5). This distribution could be partially explained by the effect that the seafloor topography exerts on the sea bottom currents around the seamount, in accordance with [27] and [28]. The bottom current fluxes approaching the rough peaks and upwelling to the top of the seamount can reach a velocity of almost twice the mean values registered along a gentle slope region with a smooth morphology [27]. As passive suspension feeders, gorgonians depend on currents for the supply of organic particles and nutrients that constitute their food source [29], [30]. Furthermore, presence of water currents also prevents suspension feeders from being buried by sediments [31].

Additionally, results for the "rock" class suggest the sponges' habitat is prevalent above small isolated rocky reliefs and along the flanks and edges of large ridges (Fig. 5). Absence of gorgonians along most of the isolated reliefs could be explained by the small scale hydrodynamic regime that characterizes these features. Since gorgonians are passive suspension feeders, they tend to settle and grow where currents flow constantly-generally along a preferential direction-ensuring a stable food supply [29]. On the other hand, the reliefs where the sponges seem to prevail, with vertical walls and irregular seafloor, may induce more complex hydrodynamic regimes with areas partially shadowed from the main flows. This habitat may not be suitable for the gorgonian assemblages but is optimal for active suspension feeder organisms such as sponges, which are able to compensate the lack of bottom currents by generating the water circulation themselves [32], [33].

Nevertheless, on-site observation is necessary to corroborate these classification results. Apart from this validation, the new information will serve as additional training data for more robust estimation of habitat distribution in the area. Other physical characteristics - such as water temperature or seabed composition - could also be incorporated to the pool of classification features, enabling for even richer description of the Chella Bank habitats. Additionally, the use of sonar images of higher resolution would provide even more insight into the different habitat classes.

\section{CONCLUSION}

The preprocessing and classification methods presented in this paper have proven to be a practical approach to the predictive habitat mapping of marine environments. This could represent a key factor in planning scientific campaigns to assess and monitor the status of living resources and facilitate policy making strategies to preserve them.

The classification method has been applied to data from the Chella seamount, showing straightforward differentiation of habitats for Gorgonian assemblages and sponges.

In the future it would be interesting to corroborate the results of the habitat classification presented in this paper by conducting specific surveys on some of the unvisited areas, thus checking the validity of the proposed habitat prediction approach.

\section{REFERENCES}

[1] C. N. Bianchi and C. Morri, "Marine biodiversity of the Mediterranean sea: Situation, problems and prospects for future research," Marine Pollution Bulletin, vol. 40, no. 5, pp. 367-376, 1996.

[2] T. P. Hughes, A. H. Baird, D. R. Bellwood, M. Card, S. R. Connolly, C. Folke, R. Grosberg, O. Hoegh-Guldberg, J. B. C. Jackson, J. Kleypas, J. M. Lough, P. Marshall, M. Nyström, S. R. Palumbi, J. M. Pandolfi, B. Rosen, and J. Roughgarden, "Climate change, human impacts, and the resilience of coral reefs," Science, vol. 301, pp. 929-933, 2003.

[3] L. Bramanti, G. Magagnini, L. De Maio, and G. Santangelo, "Recruitment, early survival and growth of the Mediterranean red coral Corallium rubrum (L 1758), a 4-year study," J. Experimental Marine Biology and Ecology, vol. 314, pp. 69-78, 2005.

[4] R. J. Beaman and P. T. Harris, "Bioregionalization of the George V shelf, east antarctica," Continental Shelf Research, vol. 25, no. 14, pp. 1657-1691, Sep. 2005.

[5] MAREMAP - UK Marine Environmental Mapping Programme [Online]. Available: http://www.noc.soton.ac.uk/shmg/maremap

[6] INFOMAR - The Integrated Mapping for the Sustainable Development of Ireland's Marine Resource [Online]. Available: http://www.infomar.ie

[7] EU Project MESH-Mapping European Seabed Habitats [Online] Available: http://www.searchmesh.net

[8] J. M. Roberts, C. J. Brown, D. Long, and C. R. Bates, “Acoustic mapping using a multibeam echosounder reveals cold-water coral reefs and surrounding habitats," in Coral Reefs. New York: Springer-Verlag, 2005, vol. 24, pp. 654-669.

[9] C. Lo Iacono, E. Gracia, S. Diez, G. Bozzano, X. Moreno, J. J. Dañobeitia, and B. Alonso, "Seafloor characterization and backscatter variability of the Almería Margin (Alboran Sea, SW Mediterranean) based on high-resolution acoustic data," Marine Geology, vol. 250, pp. 1-18, 2008.

[10] A. J. Davies, M. Wisshak, J. C. Orr, and J. M. Roberts, "Predicting suitable habitat for the cold-water coral lophelia pertusa (Scleractinia)," Deep Sea Research Part I, vol. 55, pp. 1048-1062, 2008. 
[11] D. P. Tittensor, A. R. Baco, P. E. Brewin, M. R. Clark, M. Consalvey, J. Hall-Spencer, A. A. Rowden, T. Schlacher, K. I. Stocks, and A. D. Rogers, "Predicting global habitat suitability for stony corals on seamounts," J. Biogeography, vol. 36, pp. 1111-1128, 2009.

[12] V. E. Kostylev, B. J. Todd, G. B. Fader, R. C. Courtney, G. D. Cameron, and R. A. Pickrill, "Benthic habitat mapping on the Scotian Shelf based on multibeam bathymetry, surficial geology and sea floor photographs," Marine Ecology Progress Series, vol. 219, pp. 121-137, 2001.

[13] T. L. Bryan and A. Metaxas, "Predicting suitable habitat for deepwater gorgonian corals on the Atlantic and Pacific continental margins of North America," Marine Ecology Progress Series, vol. 330, pp. 113-126, 2007.

[14] J. Guinan, C. Brown, M. F. J. Dolan, and A. J. Grehan, "Ecological niche modelling of the distribution of cold-water coral habitat using underwater remote sensing data," Ecological Informatics, vol. 4, pp. 83-92, 2009.

[15] I. Marsh and C. Brown, "Neural network classification of multibeam backscatter and bathymetry data from Stanton bank (Area IV)," Appl. Acoust., vol. 70, pp. 1269-1276, 2009.

[16] P. Blondel and O. G. Sichi, "Textural analyses of multibeam sonar imagery from stanton banks, northern ireland continental shelf," Appl. Acoust., vol. 70, pp. 1288-1297, 2009.

[17] L. Fonseca, C. Brown, B. Calder, L. Mayer, and Y. Rzhanov, "Angular range analysis of acoustic themes from stanton banks ireland: A link between visual interpretation and multibeam echosounder angular signatures," Appl. Acoust., vol. 70, pp. 1298-1304, 2009.

[18] J. Preston, "Automated acoustic seabed classification of multibeam images of Stanton Banks," Appl. Acoust., vol. 70, pp. 1277-1287, 2009.

[19] A. Muñoz, M. Ballesteros, I. Montoya, J. Ribera, J. Acosta, and E. Uchupi, “Alboran Basin, southern Spain. Part I: Geomorphology," Marine and Petroleum Geology, vol. 25, 2008.

[20] C. Lo Iacono, E. Gracia, R. Bartolome, C. Orejas, A. Gori, J. M. Gili, and J. J. Dañobeitia, "Preliminary results on the acoustic imaging of possible carbonate mounds in the Chella Bank," in Proc. 2009 GEOHAB Conf., Trondheim, Norway, May 2009.

[21] E. Coiras and D. Williams, "Approaches to automatic seabed classification book chapter," in Pattern Recognition. Rijeka, Croatia: In-Tech Publications, 2009, pp. 461-472.

[22] R. E. Bellman, Dynamic Programming. Princeton, NJ: Princeton Univ. Press, 1957.

[23] R. C. Courtney, J. T. Anderson, C. Long, and G. B. J. Fader, "Comparative seabed classification using sidescan and normal incidence sonar data at selected study sites on the Scotian shelf, Canada," in Proc. Int. Conf. Underwater Acoustic Measurements: Technology and Results, Heraklion, Crete, Jul. 2005.

[24] E. Coiras, Y. Petillot, and D. Lane, "Bathymetric side-scan Backscatter Map Restoration based on data fusion," in Proc. MTS/IEEE Oceans '03 Conf., San Diego, CA, 2003, vol. 1, pp. 287-291.

[25] W. J. Krzanowski, Principles of Multivariate Analysis. New York: Oxford Univ. Press, 1988.

[26] R. O. Duda, P. E. Hart, and D. G. Stork, Pattern Classification. New York: Wiley, 2001.

[27] A. Genin, P. K. Dayton, P. F. Lonsdale, and F. N. Spiess, "Corals on seamounts peaks provide evidence of current acceleration over deep-sea topography," Nature, vol. 322, pp. 59-61, Jul. 1986.

[28] P. M. Yoshioka and B. B. Yoshioka, "A multispecies, multiscale analysis of spatial pattern and application to a shallow-water gorgonian community," Marine Ecology Progress Series, vol. 54, pp. 257-264, 1989.

[29] J. M. Gili and R. Coma, "Benthic suspension feeders: their paramount role in littoral marine food webs," Trends in Ecology and Evolution, vol. 13, no. 8, pp. 316-321, Aug. 1998.
[30] P. B. Mortensen and L. B. Mortensen, "Distribution of deep-water gorgonian corals in relation to benthic habitat features in the Northeast Channel (Atlantic Canada)," Marine Biology, vol. 144, 2004, pp. $1223-1238$.

[31] F. Mienis, H. C. de Stigter, M. White, G. Duineveld, H. de Haas, and T. C. E. van Weering, "Hydrodynamic controls on cold-water coral growth and carbonate-mound development at the SW and SE Rockall trough Margin, NE Atlantic Ocean," Deep Sea Research Part I: Oceanographic Research Papers, vol. 54, no. 9, pp. 1655-1674, Sep. 2007.

[32] J. B. Jorgensen, "Fluid mechanical aspects of suspension feeding," Marine Ecology Progress Series, vol. 11, pp. 89-103, Feb. 1983.

[33] D. Wildish and D. Kristmanson, Benthic Suspension Feeders and Flow. Cambridge, U.K.: Cambridge Univ. Press, 1998.

Enrique Coiras received the Ph.D. degree in physics from the Complutense University of Madrid, Spain, in 1999.

From 2002 to 2006, he worked as a Research Associate at Heriot-Watt University Edinburgh, U.K., and as a software development Consultant for its spin-off company Seebyte. From 2006 to 2010, he was a Senior Research Scientist at the NATO Undersea Research Centre (NURC) in La Spezia, Italy, working on Sonar Imaging for Autonomous Underwater Robots. Since 2010, he has worked at the Capability Development division of the European Union Satellite Centre (EUSC). His current topics of research include photointerpretation, GIS, and image processing.

Claudio Lo Iacono received the Ph.D. degree in marine geology and geophysics at the University of Naples Federico II in 2004.

$\mathrm{He}$ is currently a contract researcher in the Marine Technology Unit of the Spanish National Research Council in Barcelona, Spain. His research interests include acoustic seafloor classification and habitat mapping. During the last quarter of 2010, he stayed at NURC, having obtained one of its Visiting Researcher Programme fellowships.

Eulalia Gràcia is a senior scientist at the Unidad de Tecnologia Marina (UTM), Consejo Superior de Investigaciones Cientificas (CSIC) in Barcelona, Spain. She has expertise in marine acoustics and seismics, leading research and development projects on active tectonics, geological hazards and marine paleoseismology in continental margins.

Juanjo Dañobeitia is the director of the Marine Technology Unit of the Spanish National Research Council (CSIC), having the responsibility of research vessels and polar stations to provide at national level the logistics and technological support for marine and polar research. He is also leading European and national research development projects on deep-seafloor observatories and marine geophysical sensors.

José Luis Sanz received the M.S. degree in geology from the Complutense University of Madrid, Spain.

In 1974 he joined the Spanish Oceanographic Institute (IEO) as a specialist in geology and marine cartography, working in projects related to live resources in the continental margins of Spain, Antarctica, and West Africa. He is currently involved in several European cartographic projects on bathymetry, geology, and marine habitats. 